\title{
Immunohistochemical Characteristics of Adrenocortical Carcinoma: An Overview
}

\author{
Ronald A. DeLellis ${ }^{1}$ and Sandra J. Shin ${ }^{2}$ \\ ${ }^{1}$ Department of Pathology, Lifespan Academic Medical Center and Brown University, Providence, RI, USA and ${ }^{2}$ Department \\ of Pathology, New York Presbyterian Hospital and the Weil Medical College of Cornell University, New York, NY, USA
}

Received June 12, 2003; accepted July 22, 2003

\begin{abstract}
Immunohistochemistry has played a major role in the practice of surgical pathology over the past three decades. These techniques have been of particular value in the diagnosis and classification of tumors of the adrenal cortex. The intermediate filament phenotype of adrenocortical carcinomas includes positivity for vimentin (80-100\%), cytokeratin $(40-50 \%)$ and neurofilament proteins

useful for the diagnosis of these tumors include melan A (A103), alpha inhibin, synaptophysin, calretinin, D11 and the nuclear protein adrenal 4 binding protein. The judicious use of these markers permits the distinction of adrenocortical carcinomas from other tumor types and may also be of value in the identification of the functional characteristics of these neoplasms.
\end{abstract} $(50 \%)$. Other markers that have proven

Key words: neuroendocrine, melan A, cytokeratins, inhibin, differential diagnosis

\section{Introduction}

Adrenocortical carcinomas are rare tumors with an incidence of one to two cases per million population [8]. These tumors may be associated with glucocorticoid excess (Cushing's syndrome), sex steroid overproduction (adrenogenital syndromes), mixed syndromes of glucocorticoid and sex steroid excess or mineralocorticoid overproduction. However, a significant proportion of these tumors, approaching $75 \%$ in some series, may be unassociated with syndromes of hormone excess and typically present as large retroperitoneal mass lesions [8].

The diagnosis of non-functional adrenocortical carcinomas is often challenging, particularly in cytological samples and core biopsies [10, 28] (Fig. 1). The differential diagnosis includes pheochromocytoma, renal cell carcinoma, hepatocellular carcinoma, metastatic adenocarcinoma, liposarcoma and malignant melanoma. Immunohistochemistry has become a major approach for the diagnosis of these tumors over the past two decades [7]. The purpose of this

Correspondence to: Ronald A. DeLellis, M.D., Department of Pathology, Rhode Island Hospital, 593 Eddy Street, Providence, Rhode Island, 02903, USA. brief review is to provide a conceptual framework for the immunohistochemical diagnosis of adrenocortical carcinoma.

\section{Intermediate Filaments}

The classical immunophenotype of adrenocortical carcinoma has been reported in a number of publications as showing positivity for vimentin and either negativity or equivocal positivity for cytokeratins $[5,11,30]$. However, studies of the intermediate filament composition of these tumors have produced conflicting results. Variations in immunohistochemical results for these proteins have been ascribed to the types of tissue preparation (fresh frozen vs paraffin embedded samples), fixation protocols (alcohol based vs formalin), specificities of the antibodies, use of proteolytic enzyme digestion prior to staining and the use of heat induced antigen retrieval methods.

Wick and coworkers, for example, reported positivity for vimentin in 20 of 20 adrenocortical neoplasms (10 adenomas, 10 carcinomas) following formalin fixation with or without trypsin digestion [30] (Fig. 2). In contrast, none of the cases was positive for cytokeratins without prior trypsinization while 2 of the carcinomas and 3 of the 


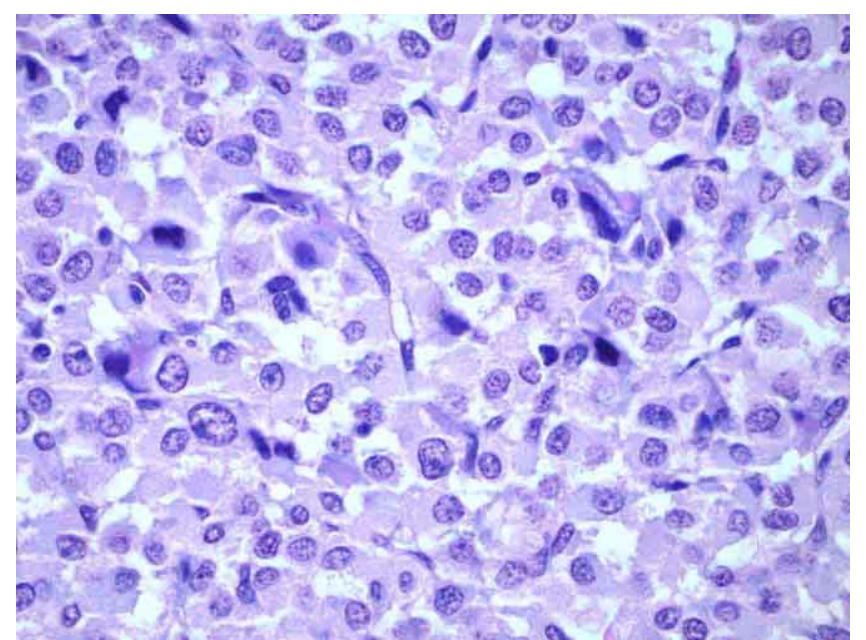

Fig. 1. Adrenocortical carcinoma (hematoxylin and eosin). The tumor shows a solid to alveolar growth pattern with a moderate degree of nuclear pleomorphism.

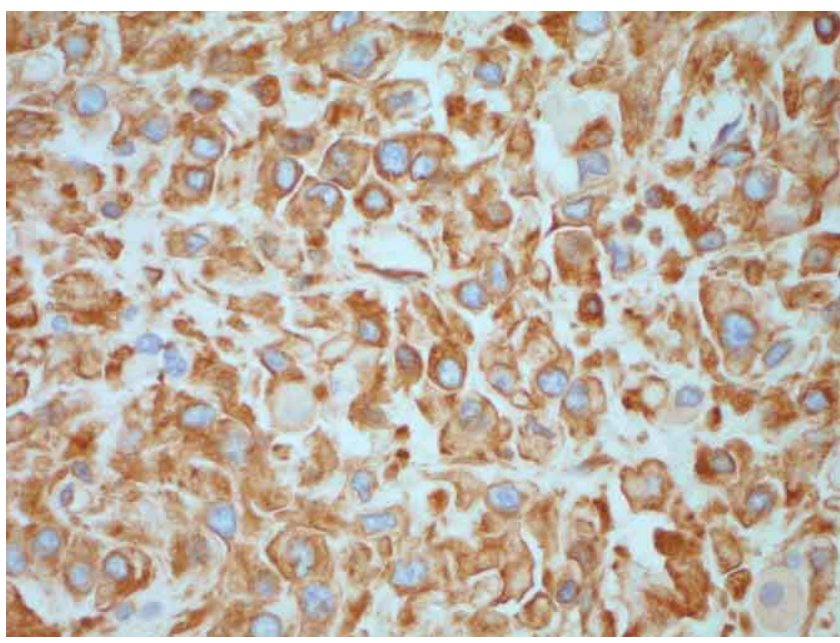

Fig. 2. Adrenocortical carcinoma (immunoperoxidase stain for vimentin following microwave induced antigen retrieval). All of the tumor cells show immunoreactivity for vimentin.

adenomas were positive following enzymatic treatment. Miettinen et al. demonstrated cytokeratins in 3 of 7 (43\%) adrenocortical carcinomas studied in alcohol fixed frozen or paraffin embedded sections and in 10 of $18(55 \%)$ formalin fixed paraffin embedded sections treated with pepsin prior to staining [20]. Subsequent studies by Miettinen established that cytokeratins 8 and 18 were the most prevalent while cytokeratins 7 and 19 were present only in trace amounts [21]. Cote and coworkers, on the other hand, were unable to demonstrate cytokeratins in 13 primary or metastatic adrenocortical carcinomas (AE1, CAM5.2) fixed in formalin and subjected to protease digestion prior to staining [5].

In a study of 31 adrenocortical carcinomas fixed in formalin and treated with trypsin, Gaffey et al. reported that only 1 case demonstrated cytokeratin immunoreactivity
[11]. Frozen sections of this tumor and an additional previously negative tumor were positive with most cytokeratin antibodies and these results were confirmed with Western blots for cytokeratins 8 and 18. The results of this study suggested that cytokeratin expression was diminished in most adrenocortical carcinomas to levels that were too low to survive the effects of formalin fixation.

The advent of microwave induced antigen retrieval techniques, however, has shown that cytokeratins 8 and 18, as demonstrated with CAM5.2, are present in at least 40$50 \%$ of adrenocortical carcinomas, and in some instances, the staining may be extensive (DeLellis, unpublished observations; Miettinen, unpublished observations) (Fig. 3). Alternatively, the staining may be focal (Fig. 4). Occasional tumors may also be positive for broad spectrum cytokeratins, as demonstrated with AE1/AE3 (Fig. 5). Interestingly, Hoang et al. have reported cytokeratins in 4 of 4 formalin fixed oncocytic adrenocortical carcinomas subjected to microwave retrieval prior to staining [13].

The results of these studies underscore the fact that a significant proportion of adrenocortical carcinomas exhibit cytokeratin immunoreactivity following the use of optimized enzyme digestion or microwave induced antigen retrieval. In addition to vimentin and cytokeratin, neurofilaments are also present in these tumors. Miettinen, for example, demonstrated immunoreactivity for the $68 \mathrm{kD}$ neurofilament subunit in 6 of 10 adrenocortical carcinomas [21]. The intermediate filament phenotype of these tumors, therefore, includes consistent positivity for vimentin, positivity for cytokeratins (predominantly 8 and 18) in 40-50\% of cases and neurofilaments in approximately $50 \%$ of cases.

\section{Neuroendocrine Markers}

In addition to neurofilament subunits, adrenocortical carcinomas may contain other markers of neuroendocrine differentiation, including synaptophysin and neuron-specific enolase [12, 18, 21]. Miettinen, for example, reported synaptophysin and neuron specific enolase in $80 \%$ and $100 \%$ of cases, respectively [21] (Fig. 6). Correlative in situ hybridization studies have confirmed the presence of synaptophysin messenger RNA in adrenocortical carcinomas [18]. Stains for chromogranin, on the other hand, are negative in adrenocortical carcinomas while pheochromocytomas typically demonstrate a synaptophysin and chromogranin positive phenotype.

\section{Melan A}

Melan A (MART-1) is a melanocyte differentiation antigen recognized by autologous T-lymphocytes $[6,7]$. This antigen can be demonstrated in formalin fixed specimens by the monoclonal antibody A103 which was produced against melan A recombinant protein. The studies of Chen et al. and Jungbluth and coworkers established the value of this antibody as a sensitive and specific marker for normal and neoplastic melanocytic cells [4, 16]. Subsequent studies 


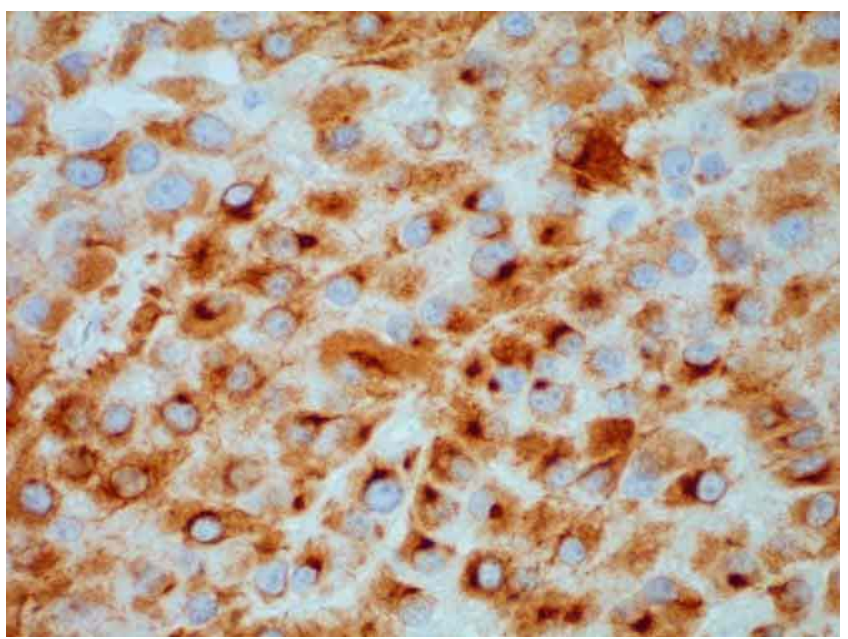

Fig. 3. Adrenocortical carcinoma (immunoperoxidase stain for cytokeratins 8 and 18 using the monoclonal antibody CAM 5.2 following microwave induced antigen retrieval). All of the tumor cells show positive staining with accentuation in the perinuclear region.

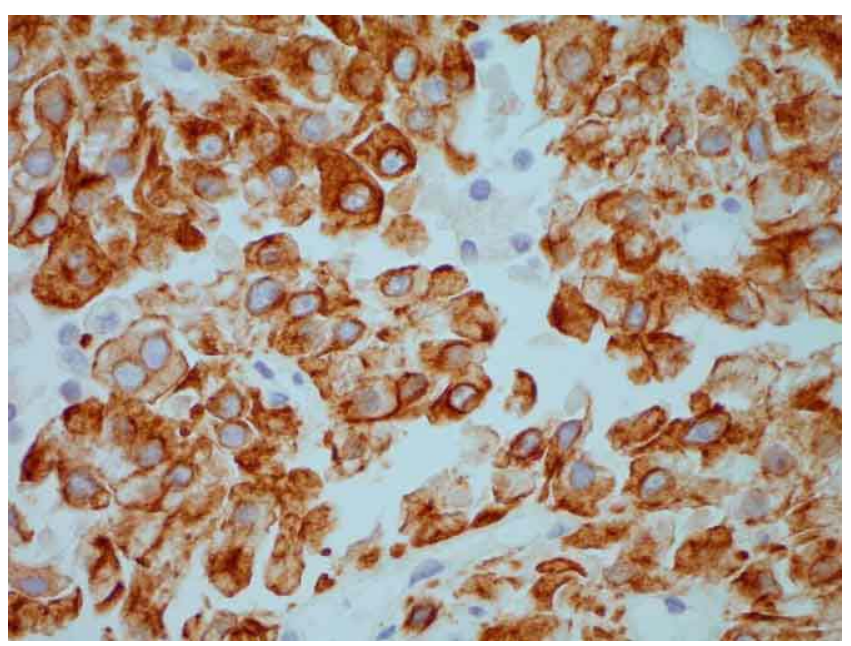

Fig. 5. Adrenocortical carcinoma (immunoperoxidase stain for broad spectrum cytokeratins using a combination of the monoclonal antibodies $\mathrm{AE} 1$ and $\mathrm{AE} 3$ following microwave induced antigen retrieval). Most of the tumor cells in this field are positive.

demonstrated that A103 immunoreactivity was also present in a limited number of other cell types including adrenocortical cells, testicular and ovarian Leydig cells, Sertoli cells, theca and granulosa cells of the ovarian follicles and luteal cells [3]. Busam and coworkers demonstrated that A103 immunoreactivity was also present in 100\% (29/29) of adrenocortical carcinomas and $100 \%(5 / 5)$ adrenocortical adenomas while pheochromocytomas, carcinomas of diverse origins and germ cell tumors were negative [3] (Fig. 7). Renshaw and Granter confirmed the utility of A103 as a marker for adrenocortical tumors but reported a lower sensitivity than that reported by Busam et al. [24]. Since that time, A103 has been reported only rarely in non-melanocytic

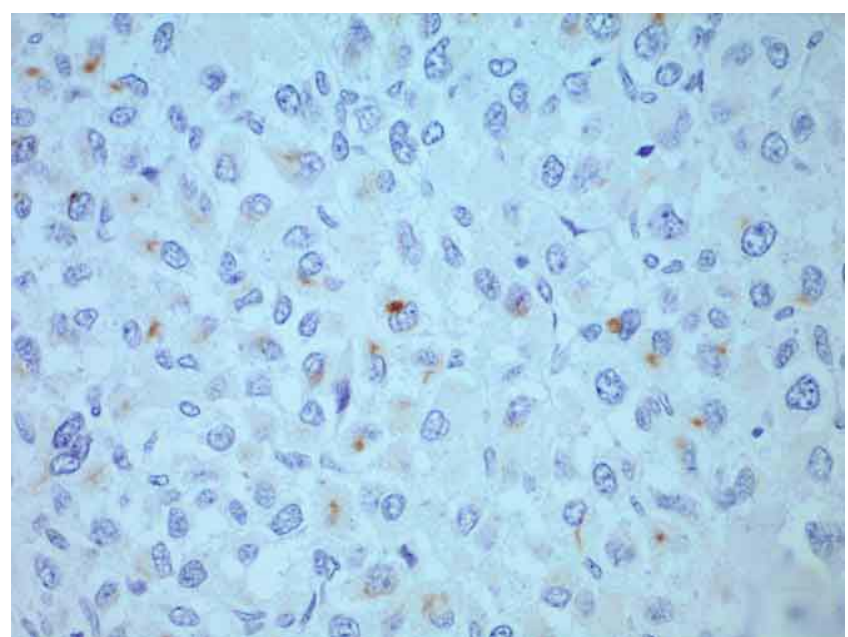

Fig. 4. Adrenocortical carcinoma (immunoperoxidase stain for cytokeratins 8 and 18 using the monoclonal antibody CAM 5.2 following microwave induced antigen retrieval). The tumor shows only focal dot-like perinuclear immunoreactivity.

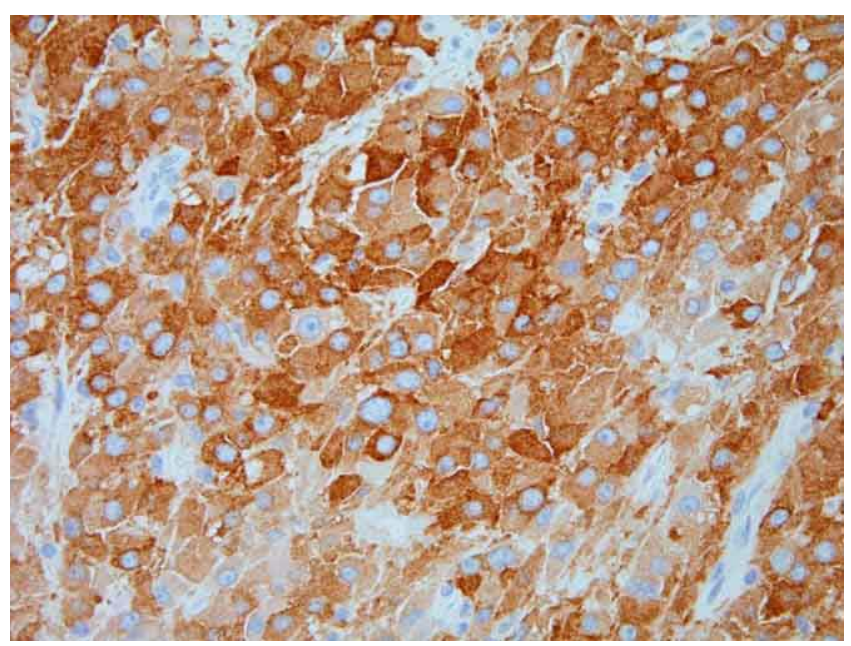

Fig. 6. Adrenocortical carcinoma (immunoperoxidase stain for synaptophysin following microwave induced antigen retrieval). All of the tumor cells show positive staining.

and non-steroid producing tumors [14]. The results of these various studies have confirmed the value of A103 as a particularly useful marker for adrenocortical carcinomas [28].

Studies utilizing reverse transcriptase PCR to demonstrate melan A mRNA in adrenal cortical tissue, however, have been unsuccessful. This observation has led to the conclusion that the immunoreactivity of A103 in cortical cells is due to a cross reaction with an epitope different from that present in melanoma cells. This conclusion is supported by the fact that other antibodies to melan A fail to react with steroid producing cells in the adrenal cortex and other sites $[4,6,28]$. 


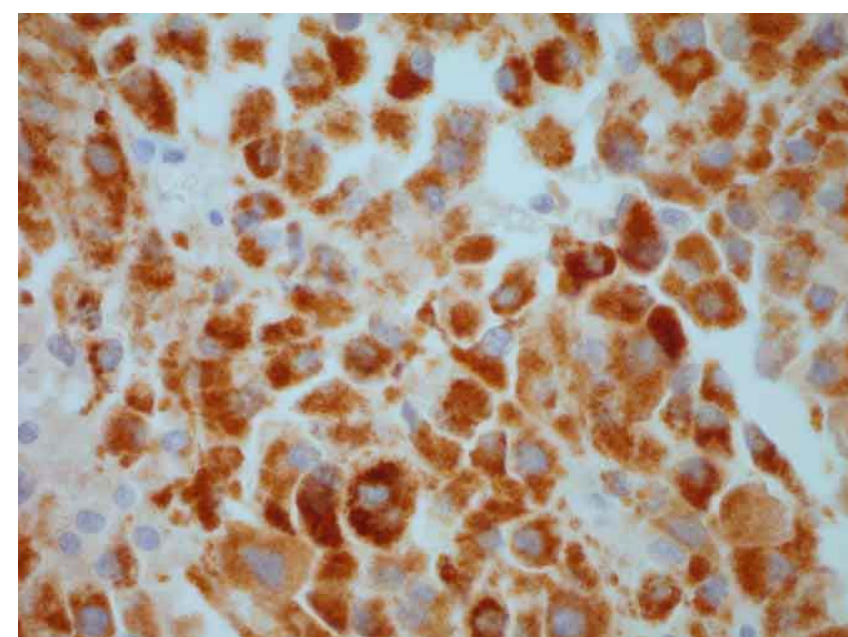

Fig. 7. Adrenocortical carcinoma (immunoperoxidase stain for melan A using the monoclonal antibody A103 following microwave induced antigen retrieval). All of the tumor cells show strong staining.

\section{Inhibins}

The inhibins are glycoprotein hormones which are present in a variety of steroid producing cells and which inhibit the secretion of follicle stimulating hormone. Immunohistochemical studies have established the utility of alpha inhibin as a sensitive and specific marker for adrenocortical carcinomas [1, 10, 23] (Fig. 8). In the study reported by Pelkey et al., alpha inhibin immunoreactivity was present in 12 of 15 (80\%) of adrenocortical carcinomas [23]. Immunoreactivity for this marker was strong in $78 \%$ of tumors associated with virilization or Cushing's syndrome while only $14 \%$ of tumors that were non-functional or associated with hyperaldosteronism were strongly reactive. Similar results have also been reported by Arola et al. [1,2].

Munro and coworkers reported positivity for alpha inhibin and beta $\mathrm{A}$ and $\mathrm{B}$ subunits in most adrenocortical tumors [22]. There were no significant differences in alpha inhibin expression between benign and malignant adrenocortical tumors although Munro et al. suggested that loss of alpha inhibin in a subgroup of carcinomas might indicate a role in tumor progression. Renshaw and Granter have compared the sensitivities and specificities of alpha inhibin and melan A (A103) and have concluded that A103 is slightly more specific and alpha inhibin slightly more sensitive for the diagnosis of adrenocortical tumors [24].

\section{Calretinin}

Calretinin is a calcium binding protein which has been used most extensively as a marker for mesotheliomas [9]. The studies of Doglioni et al. noted that this protein was present in certain steroid producing neoplasms, including ovarian stromal tumors [9]. Based on this observation, Jorda et al. have studied the distribution of calretinin in adrenal

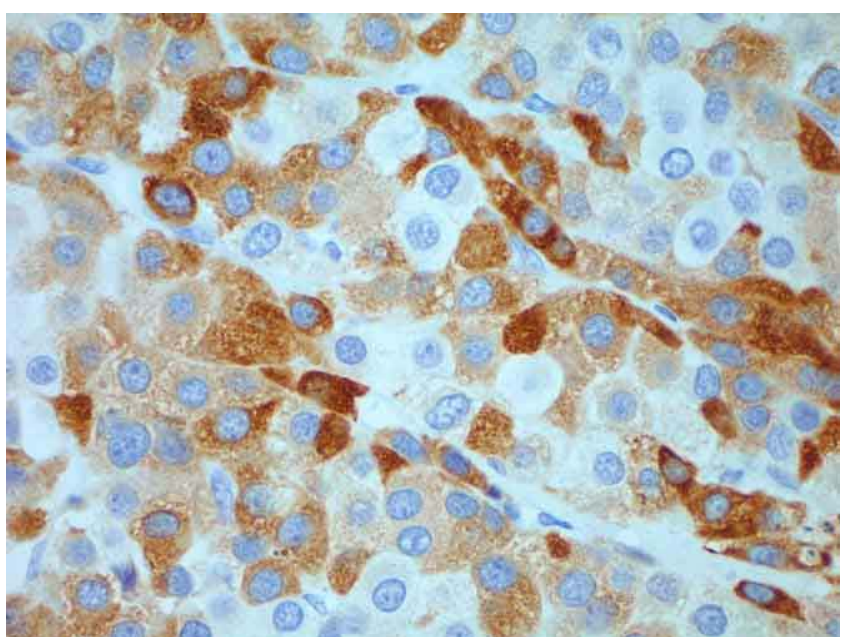

Fig. 8. Adrenocortical carcinoma (immunoperoxidase stain for inhibin A following microwave induced antigen retrieval). Some of the cells are strongly stained while others are weakly stained or negative.

cortical neoplasms [15]. In their series of adrenocortical carcinomas, immunoreactive calretinin was present in 8 of 12 adrenocortical carcinomas and 16 of 21 adenomas. The sensitivity and specificity of this marker were $73 \%$ and $100 \%$, respectively. A correlative analysis of inhibin in the same group of tumors yielded sensitivities and specificities of $70 \%$ and $100 \%$, respectively, while the combination of calretinin and inhibin yielded sensitivities and specificities of $94 \%$ and $100 \%$, respectively.

\section{Monoclonal Antibody D11}

The monoclonal antibody D11, was introduced as a marker for adrenal cortical neoplasms by Schroder et al. [27]. The antibody recognizes several $59 \mathrm{kD}$ proteins capable of binding apolipoprotein E. Schroder et al. demonstrated nuclear positivity in $100 \%$ of benign (57 cases) and malignant (15 cases) adrenocortical tumors [27]. A subsequent study by Tartour et al. demonstrated nuclear positivity in $44 \%$ ( 8 of 18 cases) of carcinomas, all of which were well differentiated [29]. The staining pattern was focal (less than 20\%) in 2 of 8 cases and diffuse in the remaining 6 cases. Nuclear staining was accompanied by varying degrees of cytoplasmic staining. Four additional cases showed cytoplasmic staining without nuclear staining while 6 cases were entirely negative. Cytoplasmic staining for D11, however, is not specific for adrenocortical carcinomas since it was also present in 5/18 (27.7\%) of metastases to the adrenals, $20 / 20(100 \%)$ of hepatocellular carcinomas, $3 / 5(60 \%)$ of lung carcinomas and $1 / 50(2 \%)$ of renal cell carcinomas [29]. Nuclear reactivity for D11, therefore, has a high specificity but relatively low sensitivity for the diagnosis of adrenocortical carcinomas. In a more recent study, Komminoth et al. reported D11 immunoreactivity in $22 / 27(81 \%)$ of adrenocortical carcinomas [18]. 
Table 1. Differential diagnosis of adrenal cortical carcinoma

\begin{tabular}{|c|c|c|c|c|c|c|c|c|c|c|c|c|c|c|c|}
\hline & $\mathrm{CK}$ & VIM & EMA & CEA & $\mathrm{S} 100$ & HBM45 & MEL & NF & SYN & CGA & AFP & CAL & INH & $\mathrm{RCC}$ & HEPPAR-1 \\
\hline Adrenal Cortical Ca & $+1-$ & + & - & - & $-1+$ & - & + & + & + & - & - & + & + & - & - \\
\hline Pheochromocytoma & - & $+/-$ & - & - & + & $-1+$ & - & + & + & + & - & - & - & - & - \\
\hline Renal Cell Ca & + & + & + & - & $+/-$ & - & - & - & - & - & - & $-/+$ & $-1+$ & + & - \\
\hline Hepatocellular $\mathrm{Ca}$ & + & + & + & + & $+/-$ & - & - & - & - & - & + & $-/+$ & $-1+$ & - & + \\
\hline Metastatic Adeno Ca & + & $+/-$ & + & + & $+/-$ & - & - & - & - & - & - & $-/+$ & - & - & - \\
\hline Liposarcoma & - & + & - & - & + & - & $-1+$ & - & - & - & - & - & - & - & - \\
\hline Malignant melanoma & $-/+$ & + & - & - & + & + & + & - & - & - & - & - & - & - & - \\
\hline
\end{tabular}

Key: +, positive; +/-, predominantly positive; -/+, predominantly negative; - , negative.

CK, cytokeratin (CAM 5.2); VIM, vimentin; EMA, epithelial membrane antigen; MEL, melan A (A103); NF, neurofilament; SYN, synaptophysin; CGA, chromogranin A; AFP, alpha fetoprotein; CAL, calretinin; INH, inhibin A; RCC, renal cell carcinoma antigen; HEPPAR-1, hepatocyte specific antibody.

\section{Transcription Factors and Steroidogenic Enzymes}

The nuclear protein adrenal 4 binding protein (Ad4BP) or steroid factor 1 (SF-1) is a transcription factor that has also been used for the diagnosis of adrenocortical carcinomas [26]. This marker has been demonstrated in $8 / 8(100 \%)$ of adrenocortical carcinomas but is absent from hepatocellular carcinomas, melanomas, ovarian and uterine clear cell carcinomas, large cell bronchogenic carcinomas and pheochromocytomas. Ad4BP has also been reported in Leydig cells of the testis, thecal and granulosa cells of the ovary and gonadotropin producing cells of the adenohypophysis.

Antibodies to steroidogenic hormones have also been used in immunohistochemical formats for the study of adrenocortical carcinomas. Sasano et al., for example, have demonstrated that a number of cells in adrenocortical carcinomas did not express all of the enzymes required for the synthesis of biologically active steroids [25]. This observation may, in fact, be responsible for the fact that increased levels of precursor steroid are present in adrenocortical carcinomas. However, there have been relatively few applications of immunohistochemical methods for steroidogenic enzymes in the diagnostic setting.

\section{Other Markers}

A variety of other markers have been employed to differentiate adrenocortical carcinomas from other tumor types (Table 1). For example, most renal cell carcinomas express Lewis blood group isoantigens (e.g. Lewis X) while adrenocortical carcinomas do not [5]. Most adenocarcinomas are positive for carcinoembryonic antigen, epithelial membrane antigen and CD15 while adrenocortical carcinomas are negative [7, 27]. Adrenocortical carcinomas are also negative with the renal cell carcinoma (RCC) and hepatocyte antibodies [19, 31] (Table 1).

\section{References}

1. Arola, J., Liu, J., Heikkila, P., Voutilainen, R. and Kahri, A. (1998) Expression of inhibin alpha in the human adrenal gland and adrenocortical tumors. Endocr. Res. 24; 865-867.

2. Arola, J., Liu, J., Heikkila, P., Ilvesmaki, V., Salmenkivi, K., Voutilainen, R. and Kahri, A. I. (2000) Expression of inhibin alpha in adrenocortical tumours reflects the hormonal status of the neoplasm. J. Endocrinol. 165; 223-229.

3. Busam, K. J., Iversen, K., Coplan, K. A., Old, L. J., Stockert, E., Chen, Y. T., McGregor, D. and Jungbluth, A. (1998) Immunoreactivity for A103, an antibody to melan-A (Mart-1), in adrenocortical and other steroid tumors. Am. J. Surg. Pathol. 22; 57-63.

4. Chen, Y. T., Stockert, E., Jungbluth, A., Tsang, S., Coplan, K. A., Scanlan, M. J. and Old, L. J. (1996) Serological analysis of Melan-A (MART-1), a melanocyte-specific protein homogeneously expressed in human melanomas. Proc. Natl. Acad. Sci. U S A 93; 5915-5919.

5. Cote, R. J., Cardon-Cardo, C., Reuter, V. E. and Rosen, P. P. (1990) Immunopathology of adrenal and renal cortical tumors: Coordinated change in antigen expression is associated with neoplastic conversion in the adrenal cortex. Am. J. Pathol. 136; $1077-1084$.

6. Coulie, P. G., Brichard, V., Van Pel, A., Wolfel, T., Schneider, J., Traversari, C., Mattei, S., De Plaen, E., Lurquin, C., Szikora, J. P., et al. (1994) A new gene coding for a differentiation antigen recognized by autologous cytolytic T lymphocytes on HLA-A2 melanomas. J. Exp. Med. 180; 35-42.

7. DeLellis, R. A. and Shin, S. J. (2002) Diagnostic immunohistochemistry of endocrine tumors. In "Diagnostic Immunohistochemistry", ed. by D. J. Dabbs, Churchill Livingstone, New York, pp. 209-240.

8. DeLellis, R. A. (1999) The adrenal glands. In "Diagnostic Surgical Pathology", 3rd ed., ed. by S. S. Sternberg and D. A. Antonioli, Lippincott, Williams and Wilkins, Philadelphia, pp. 589-624.

9. Doglioni, C., Tos, A. P., Laurino, L., Iuzzolino, P., Chiarelli, C., Celio, M. R. and Viale, G. (1996) Calretinin: a novel immunocytochemical marker for mesothelioma. Am. J. Surg. Pathol. 20; 1037-1046.

10. Fetsch, P. A., Powers, C. N., Zakowski, M. F. and Abati, A. (1999) Anti-alpha-inhibin: marker of choice for the consistent distinction between adrenocortical carcinoma and renal cell carcinoma in fine needle aspiration. Cancer $87 ; 168-172$.

11. Gaffey, M. J., Traweek, S. T., Mills, S. E., Travis, W. D., Lack, 
E. E., Medeiros, L. J. and Weiss, L. M. (1992) Cytokeratin expression in adrenocortical neoplasia: an immunohistochemical and biochemical study with implications for the differential diagnosis of adrenocortical, hepatocellular, and renal cell carcinoma. Hum. Pathol. 23; 144-153.

12. Haak, H. R. and Fleuren, G.-J. (1995) Neuroendocrine differentiation of adrenocortical tumors. Cancer 75; 860-864.

13. Hoang, M. P., Ayala, A. G. and Albores-Saavedra, J. (2002) Oncocytic adrenocortical carcinoma: A morphologic, immunohistochemical and ultrastructural study of four cases. Mod. Pathol. 15; 973-978.

14. Huang, H. Y. and Antonescu, C. (2002) Epithelioid variant of pleomorphic liposarcoma: a comparative immunohistochemical and ultrastructural analysis of six cases with emphasis on overlapping features with epithelial malignancies. Ultrastruct. Pathol. $26 ; 299-308$

15. Jorda, M., De, M. B. and Nadji, M. (2002) Calretinin and inhibin are useful in separating adrenocortical neoplasm from pheochromocytomas. Appl. Immunohistochem. Mol. Morphol. 10; 67-70.

16. Jungbluth, A. A., Busam, K. J., Gerald, W. L., Stockert, E., Coplan, K. A., Iversen, K., MacGregor, D. P., Old, L. J. and Chen, Y. T. (1998) A103: An anti-melan-a monoclonal antibody for the detection of malignant melanoma in paraffin-embedded tissues. Am. J. Surg. Pathol. 22; 595-602.

17. Kawakami, Y., Eliyahu, S., Delgado, C. H., Robbins, P. F., Rivoltini, L., Topalian, S. L., Miki, T. and Rosenberg, S. A (1994) Cloning of the gene coding for a shared human melanoma antigen recognized by autologous $\mathrm{T}$ cells infiltrating into tumor. Proc. Natl. Acad. Sci. U S A 91; 3515-3519.

18. Komminoth, P., Roth, J., Schroder, S., Saremaslani, P. and Heitz, P. U. (1995) Overlapping expression of immunohistochemical markers and synaptophysin mRNA in pheochromocytomas and adrenocortical carcinomas. Implications for the differential diagnosis of adrenal gland tumors. Lab. Invest. 72; 424-431.

19. McGregor, D. K., Khurana, K. K., Cao, C., Tsao, C. C., Ayala, G., Krishnan, B., Ro, J. Y., Lechago, J. and Truong, L. D. (2001) Diagnosing primary and metastatic renal cell carcinoma: the use of the monoclonal antibody 'Renal Cell Carcinoma Marker'. Am. J. Surg. Pathol. 25; 1485-1492.

20. Miettinen, M., Lehto, V. P. and Virtanen, I. (1985) Immunofluorescence microscopic evaluation of the intermediate filament expression of the adrenal cortex and medulla and their tumors. Am. J. Pathol. 118; 360-366.

21. Miettinen, M. (1992) Neuroendocrine differentiation in adreno- cortical carcinoma. New immunohistochemical findings supported by electron microscopy. Lab. Invest. 66; 169-174.

22. Munro, L. M., Kennedy, A. and McNicol, A. M. (1999) The expression of inhibin/activin subunits in the human adrenal cortex and its tumors. J. Endocrinol. 161; 341-347.

23. Pelkey, T. J., Frierson, H. F., Mills, S. E. and Stoler, M. H. (1998) The alpha subunit of inhibin in adrenal cortical neoplasia. Mod. Pathol. 11; 516-524.

24. Renshaw, A. A. and Granter, S. R. (1998) A comparison of A103 and inhibin reactivity in adrenal cortical tumors: Distinction from hepatocellular carcinoma and renal tumors. Mod. Pathol. 11; $1160-1164$.

25. Sasano, H., Suzuki, T., Nagura, H. and Nishikawa, T. (1993) Steroidogenesis in human adrenocortical carcinoma. Biochemical activities, immunohistochemistry, and in situ hybridization of steroidogenic enzymes and histopathological study in nine cases. Hum. Pathol. 24; 397-404.

26. Sasano, H., Shizawa, S., Suzuki, T., Takayama, K., Fukaya, T., Morohashi, K. and Nagura, H. (1995) Transcription factor adrenal 4 binding protein as a marker of adrenocortical malignancy. Hum. Pathol. 26; 1154-1156.

27. Schroder, S., Padberg, B. C., Achilles, E., Holl, K., Dralle, H. and Kloppel, G. (1992) Immunocytochemistry in adrenocortical tumours: a clinicomorphological study of 72 neoplasms. Virchows Arch. A Pathol. Anat. Histopathol. 420; 65-70.

28. Shin, S. J., Hoda, R. S., Ying, L. and DeLellis, R. A. (2000) Diagnostic utility of the monoclonal antibody A103 in fine needle aspiration biopsies of the adrenal. Am. J. Clin. Pathol. 113; 295302.

29. Tartour, E., Caillou, B., Tenenbaum, F., Schroder, S., Luciani, S., Talbot, M. and Schlumberger, M. (1993) Immunohistochemical study of adrenocortical carcinoma. Predictive value of the D11 monoclonal antibody. Cancer 72; 3296-3303.

30. Wick, M. R., Cherwitz, D. L., McGlennen, R. C. and Dehner, L. P. (1986) Adrenocortical carcinoma: An immunohistochemical comparison with renal cell carcinoma. Am. J. Pathol. 122; 343352.

31. Wieczorek, T. J., Pinkus, J. L., Glickman, J. N. and Pinkus, G. S. (2002) Comparison of thyroid transcription factor-1 and hepatocyte antigen immunohistochemical analysis in the differential diagnosis of hepatocellular carcinoma, metastatic adenocarcinoma, renal cell carcinoma, and adrenal cortical carcinoma. Am. J. Clin. Pathol. 118; 911-921. 